\title{
THE NATURE OF THE HIGH VELOCITY FLOW IN CRL 618
}

\author{
R.NERI, M.GUÉLIN, S.GUILLOTEAU and R.LUCAS \\ I.R.A.M, Domaine Universitaire, 300 rue de la Piscine, F - 38406 St-Martin-d'Hères \\ and \\ S.GARCIA-BURILLO and J.CERNICHARO \\ C.A.Y. - I.G.N., S - 19080 Guadalajara
}

Using the IRAM interferometer, we have mapped with a 2 ". $4 \times 3$ ". 4 resolution the $J=1 \rightarrow 0 \mathrm{HCN}$ line emission in the proto-planetary nebula CRL 618. Our maps resolve the $200 \mathrm{kms}^{-1}$ molecular outflow (Cernicharo et al. 1989), as well as the slowly expanding circumstellar envelope (Bujarrabal et al. 1988), allowing a very precise positioning $\left(\leq 0^{\prime \prime} 1\right)$ of these components with respect to the central HII region. $70 \%$ of the $\mathrm{HCN}$ envelope emission comes from a very compact, spherically symmetric core of size $\simeq 3$ '! 2 . The core surrounds the high velocity gas which appears localized in a number of small 'clumps' $(\leq 0$ ' 5$)$ - see figure. The large range of velocities observed in the 'clumps' suggests that we are not observing a decelerating molecular flow, but the impacts of a bipolar outflow on the slowly moving core, close to the HII region. The collision of a neutral gas outflow with high density regions (the 'clumps') results in the generation of dissociative shock-waves pushing and tearing the inner surface of the envelope. CRL 618 appears to have reached the stage where the stellar winds begin to disrupt and to scrape through the massive envelope, shortly before it evolves towards a Planetary Nebula.

The molecules have certainly been destroyed by the fast shock before the gas was accelerated to $200 \mathrm{kms}^{-1}$. Expansion and cooling of the accelerated gas in the post-shock region allow their reformation (see the insert). We infer from the observed geometry and velocities that $\mathrm{HCN}$ reformation occurs in $\leq 50 \mathrm{y}$. The small size of the HCN high velocity sources implies large excitation temperatures $(200 \mathrm{~K}$ $\left.\leq T_{e x} \leq 1000 \mathrm{~K}\right)$ and a large $\mathrm{HCN}$ abundance $x(\mathrm{HCN}) / x(\mathrm{CO}) \geq 0.1$. This is the first time that the HCN abundance and formation time scale have been measured behind a fast shock. These results will be presented by Neri et al. 1992 .

Figure: Morphology of CRL 618's core, as derived from the HCN data. The figure width is $\simeq 4^{\prime \prime}$.

\section{References}

Bujarrabal et al.: 1988, 'PPN: the case of CRL 618', A\&A, 204, 242

Cernicharo et al.: 1989, $A \mathscr{E} A, \mathbf{2 2 2}, \mathrm{L} 1$

Neri et al.: 1992, 'CRL618: The nature of the $200 \mathrm{kms}^{-1}$ outflow', $A \mathscr{E} A$, in press

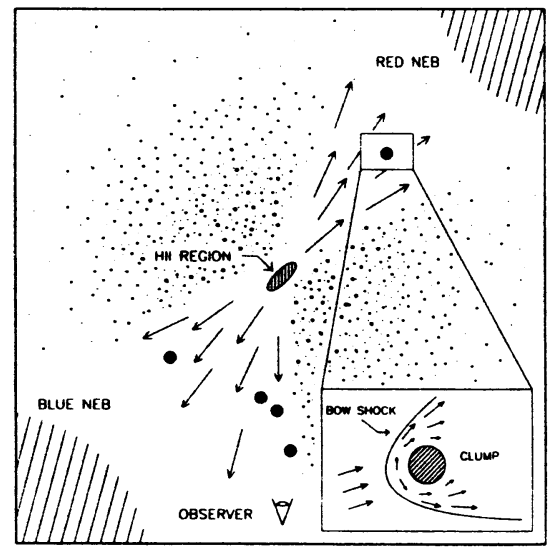

